\title{
TRITERPENÓIDES PENTACÍCLICOS DAS FOLHAS DE Terminalia brasiliensis
}

\author{
Delton Sérvulo Araújo e Mariana H. Chaves* \\ Departamento de Química, Universidade Federal do Piauí, 64049-550 Teresina - PI
}

Recebido em 8/9/04; aceito em 15/2/05; publicado na web em 10/8/05

\begin{abstract}
PENTACYCLIC TRITERPENOIDS FROM THE LEAVES OF Terminalia brasiliensis. Eleven oleanane, ursane and lupane-type

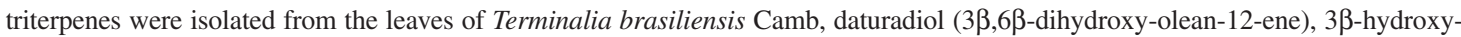
30-norlupan-20-one, lupenone, $\beta$-amyrenone, $\alpha$-amyrenone, lupeol, $\beta$-amyrin, $\alpha$-amyrin, betulin, erythrodiol and uvaol, in addition to squalene, sitosterol and $\alpha$-tocopherol. The structures of these compounds were identified by ${ }^{1} \mathrm{H}$ and ${ }^{13} \mathrm{C}$ NMR spectral analysis and comparison with literature data.
\end{abstract}

Keywords: Terminalia brasiliensis; triterpene; ${ }^{13} \mathrm{C}$ NMR data.

\section{INTRODUÇÃO}

A família Combretaceae é constituída por aproximadamente 600 espécies. Os dois gêneros de maior ocorrência são Combretum e Terminalia, cada um com 250 espécies, sendo extensamente usadas na medicina tradicional da África ${ }^{1}$.

As plantas do gênero Terminalia são amplamente distribuídas nas áreas tropicais do mundo e conhecidas como uma fonte rica de metabólitos secundários, tais como triterpenóides pentacíclicos e seus derivados glicosilados, flavonóides, taninos e outros compostos $\operatorname{aromáticos}^{1,2}$.

As espécies do gênero Terminalia apresentam diversas atividades farmacológicas reveladas pelos extratos ou substâncias isoladas, tais como antifúngica, antimicrobiana, antioxidante, antidiabética, anti-HIV-1, antimalárica e outras ${ }^{3}$.

Terminalia brasiliensis Camb. é conhecida popularmente como amêndoa-brava, cerne-amarelo, capitão-do-campo, catinga-de-porco, mussambé, entre outros. Sua utilização é amplamente difundida, sendo usada na medicina popular no tratamento de "barriga inchada e disenteria"; fornece madeira de lei, para construção civil, marcenaria e carpintaria; produz matéria tintorial e a casca é escura e adequada para utilização em curtume ${ }^{4}$.

Este artigo relata os primeiros resultados do estudo fitoquímico das folhas de um espécimen de T. brasiliensis, descrevendo o isolamento e identificação estrutural de doze triterpenóides, destes, cinco foram obtidos puros: daturadiol [3 $3,6 \beta$-diidroxi-olean-12eno (1)], 3ß-hidroxi-30-norlupan-20-ona (2), lupenona (3c), betulina (5c) e esqualeno (6) e os demais subdivididos em três misturas: a primeira constituída pela $\beta$-amirenona (3a), $\alpha$-amirenona (3b), lupenona (3c); a segunda pela $\beta$-amirina (4a), $\alpha$-amirina (4b) e lupeol (4c) e a terceira pelo eritrodiol (5a), uvaol (5b) e betulina (5c). Além dos triterpenos, foram isolados o sitosterol (7) e o $\alpha$ tocoferol (8). Os triterpenóides $\mathbf{1}$ e $\mathbf{2}$ estão sendo relatados pela primeira vez na família Combretaceae e as substâncias $\mathbf{3 a}, \mathbf{3 b}, \mathbf{4 b}$, $\mathbf{5 a}, \mathbf{5 b}$ e $\mathbf{8}$ no gênero Terminalia.

\section{RESULTADOS E DISCUSSÃO}

O fracionamento cromatográfico do material solúvel em metanol, proveniente da fase hexânica do extrato etanólico das fo- lhas, conduziu ao isolamento de 12 triterpenóides, um esteróide e um tocoferol (Figura 1). As substâncias, 3a-3c, 4a-4c, 5a-5c, 6, 7 e 8 são comuns em outras espécies e foram identificadas através da análise dos dados espectrais de $\mathrm{RMN}{ }^{1} \mathrm{H}$ e ${ }^{13} \mathrm{C}$ e comparação com os registrados na literatura ${ }^{5-8}$. Os triterpenóides $\mathbf{1}$ e $\mathbf{2}$ foram identificados utilizando também dados dos espectros de RMN 2D.

O triterpenóide 1, um raro derivado olean-12-eno denominado daturadiol, foi relatado somente nas espécies Datura innoxia Mill. e Datura stramonium L. pertencentes à família Solanaceae e como

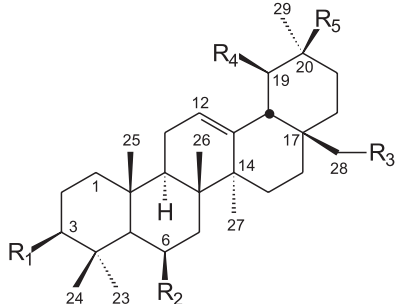

1: $\mathrm{R}_{1}=\mathrm{R}_{2}=\mathrm{OH}, \mathrm{R}_{3}=\mathrm{R}_{4}=\mathrm{H}, \mathrm{R}_{5}=\mathrm{CH}_{3}$

3a: $\mathrm{R}_{1}=\mathrm{O}, \mathrm{R}_{2}=\mathrm{R}_{3}=\mathrm{R}_{4}=\mathrm{H}, \mathrm{R}_{5}=\mathrm{CH}_{3}$

3b: $\mathrm{R}_{1}=\mathrm{O}, \mathrm{R}_{2}=\mathrm{R}_{3}=\mathrm{R}_{5}=\mathrm{H}, \mathrm{R}_{4}=\mathrm{CH}_{3}$

4a: $\mathrm{R}_{1}=\mathrm{OH}, \mathrm{R}_{2}=\mathrm{R}_{3}=\mathrm{R}_{4}=\mathrm{H}, \mathrm{R}_{5}=\mathrm{CH}_{3}$

4b: $\mathrm{R}_{1}=\mathrm{OH}, \mathrm{R}_{2}=\mathrm{R}_{3}=\mathrm{R}_{5}=\mathrm{H}, \mathrm{R}_{4}=\mathrm{CH}_{3}$

5a: $\mathrm{R}_{1}=\mathrm{R}_{3}=\mathrm{OH}, \mathrm{R}_{2}=\mathrm{R}_{4}=\mathrm{H}, \mathrm{R}_{5}=\mathrm{CH}_{3}$

5b: $\mathrm{R}_{1}=\mathrm{R}_{3}=\mathrm{OH}, \mathrm{R}_{2}=\mathrm{R}_{5}=\mathrm{H}, \mathrm{R}_{4}=\mathrm{CH}_{3}$

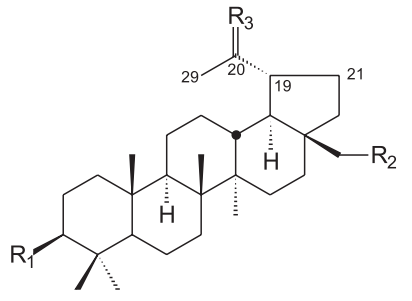

2: $\mathrm{R}_{1}=\mathrm{OH}, \mathrm{R}_{2}=\mathrm{H}, \mathrm{R}_{3}=\mathrm{O}$

3c: $\mathrm{R}_{1}=\mathrm{O}, \mathrm{R}_{2}=\mathrm{H}, \mathrm{R}_{3}=\mathrm{CH}_{2}$

4c: $\mathrm{R}_{1}=\mathrm{OH}, \mathrm{R}_{2}=\mathrm{H}, \mathrm{R}_{3}=\mathrm{CH}_{2}$

5c: $\mathrm{R}_{1}=\mathrm{R}_{2}=\mathrm{OH}, \mathrm{R}_{3}=\mathrm{CH}_{2}$

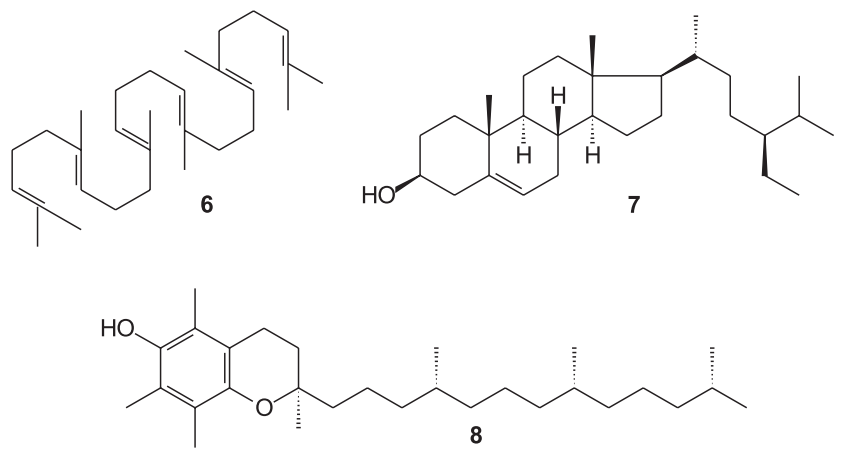

Figura 1. Substâncias isoladas de Terminalia brasiliensis 
derivado sintético, no entanto seus dados de $\mathrm{RMN}{ }^{13} \mathrm{C}$ não foram descritos ${ }^{9}$.

O espectro de RMN ${ }^{1} \mathrm{H}$ da substância 1 (Tabela 1) apresentou sete singletos na região entre $\delta$ 0,80-1,40 referentes aos hidrogênios de grupos metílicos, sendo o sinal em $\delta$ 0,88 atribuído aos hidrogênios de dois grupos $\mathrm{CH}_{3}(\mathrm{H}-29$ e H-30) e um duplo-dubleto em $\delta 3,17(1 \mathrm{H}, \mathrm{J}=5$ e $10 \mathrm{~Hz})$, característico de hidrogênio carbinólico em triterpenos $3 \beta-\mathrm{OH}$. O espectro apresentou, ainda, um sinal em $\delta 4,58$ com integração correspondente a um hidrogênio, sugerindo a existência de uma segunda hidroxila e um dubleto em $\delta 5,24(1 \mathrm{H}$, $\mathrm{J}=3 \mathrm{~Hz}$ ), característico de hidrogênio olefínico.

$\mathrm{O}$ espectro de RMN ${ }^{13} \mathrm{C}$ BB- ${ }^{1} \mathrm{HD}$ de $\mathbf{1}$ (Tabela 1) mostrou 29 sinais, um com intensidade relativa a dois carbonos $(\delta 17,0)$ e, pelo espectro DEPT $135^{\circ}$, oito são de carbonos metílicos, nove metilênicos, seis metínicos e sete não-hidrogenados, correspondendo à fórmula molecular $\mathrm{C}_{30} \mathrm{H}_{50} \mathrm{O}_{2}$. A região de olefinas deste espectro apresentou sinais em $\delta 122,0(\mathrm{CH})$ e 144,3 (C), que definiram o esqueleto olean-12-eno 5 . O sinal em $\delta 79,1(\mathrm{CH})$ é característico de C-3 em triterpenóides $3 \beta-\mathrm{OH}$ e o sinal em $\delta 68,7(\mathrm{CH})$

Tabela 1. Dados de $\mathrm{RMN}{ }^{1} \mathrm{H}(500 \mathrm{MHz})$ e ${ }^{13} \mathrm{C}(125 \mathrm{MHz})$ de 1, em $\mathrm{CDCl}_{3}$

\begin{tabular}{|c|c|c|c|}
\hline $\mathrm{C} / \mathrm{H}$ & $\delta_{\mathrm{C}}$ & $* \delta_{\mathrm{H}}$ & ${ }^{1} \mathrm{H} \times{ }^{13} \mathrm{C}-\mathrm{HMBC}-{ }^{\mathrm{n}} \boldsymbol{J}_{\mathrm{CH}}(\mathrm{n}=2$ e 3$)$ \\
\hline 1 & 40,7 & $1,60,1,78$ & $\mathrm{H}-5, \mathrm{H}-25\left({ }^{3} J_{\mathrm{CH}}\right)$ \\
\hline 2 & 27,4 & 1,67 & $\mathrm{H}-1\left({ }^{2} J_{\mathrm{CH}}\right)$ \\
\hline 3 & 79,1 & $3,17(\mathrm{dd}, \mathrm{J}=5,10)$ & $\begin{array}{c}\mathrm{H}-2\left({ }^{2} J_{\mathrm{CH}}\right) ; \mathrm{H}-1, \mathrm{H}-5, \mathrm{H}-23, \\
\mathrm{H}-24\left({ }^{3} J_{\mathrm{CH}}\right)\end{array}$ \\
\hline 4 & 39,6 & - & H-5, H-23, H-24 $\left({ }^{2} J_{\mathrm{CH}}\right)$ \\
\hline 5 & 55,6 & 0,76 & $\mathrm{H}-7, \mathrm{H}-23, \mathrm{H}-24, \mathrm{H}-25\left({ }^{3} J_{\mathrm{CH}}\right)$ \\
\hline 6 & 68,7 & $4,58\left(\mathrm{sl}, W_{\mathrm{h} / 2}=9\right)$ & H-5, H-7 $\left({ }^{2} J_{\mathrm{CH}}\right)$ \\
\hline 7 & 40,8 & $0,97,1,54$ & H-26 $\left({ }^{3} J_{\mathrm{CH}}\right)$ \\
\hline 8 & 38,9 & - & H-9 $\left({ }^{2} J_{\mathrm{CH}}\right) ; \mathrm{H}-11, \mathrm{H}-15\left({ }^{3} J_{\mathrm{CH}}\right)$ \\
\hline 9 & 47,9 & 1,59 & $\begin{array}{c}\mathrm{H}-11\left({ }^{2} J_{\mathrm{CH}}\right) ; \mathrm{H}-7, \mathrm{H}-25, \\
\mathrm{H}-26\left({ }^{3} J_{\mathrm{CH}}\right)\end{array}$ \\
\hline 10 & 36,4 & - & H-1, H-5, H-9, H-25 $\left({ }^{2} J_{\mathrm{CH}}\right)$ \\
\hline 11 & 23,4 & $1,90,2,01$ & H-9 $\left({ }^{2} J_{\mathrm{CH}}\right)$ \\
\hline 12 & 122,0 & $5,24(\mathrm{~d}, \mathrm{~J}=3)$ & H-11 $\left({ }^{2} J_{\mathrm{CH}}\right) ; \mathrm{H}-9, \mathrm{H}-18\left({ }^{3} J_{\mathrm{CH}}\right)$ \\
\hline 13 & 144,3 & - & $\begin{array}{c}\mathrm{H}-18\left({ }^{2} J_{\mathrm{CH}}\right) ; \mathrm{H}-11, \mathrm{H}-19, \\
\mathrm{H}-27\left({ }^{3} J_{\mathrm{CH}}\right)\end{array}$ \\
\hline 14 & 42,3 & - & $\begin{array}{c}\mathrm{H}-15, \mathrm{H}-27\left({ }^{2} J_{\mathrm{CH}}\right) ; \mathrm{H}-9, \\
\mathrm{H}-26\left({ }^{3} J_{\mathrm{CH}}\right)\end{array}$ \\
\hline 15 & 26,1 & $0,94,1,80$ & $\mathrm{H}-27\left({ }^{3} J_{\mathrm{CH}}\right)$ \\
\hline 16 & 27,0 & $0,81,1,95$ & $\mathrm{H}-28\left({ }^{3} J_{\mathrm{CH}}\right)$ \\
\hline 17 & 32,5 & - & H-18, H-28 $\left({ }^{2} J_{\mathrm{CH}}\right)$ \\
\hline 18 & 47,2 & 1,98 & H-19 $\left({ }^{2} J_{\mathrm{CH}}\right) ; \mathrm{H}-28\left({ }^{3} J_{\mathrm{CH}}\right)$ \\
\hline 19 & 46,8 & $1,04,1,64$ & H-29, H-30 $\left({ }^{3} J_{\mathrm{CH}}\right)$ \\
\hline 20 & 31,4 & - & H-19, H-29, H-30 $\left({ }^{2} J_{\mathrm{CH}}\right)$ \\
\hline 21 & 34,7 & $1,33,1,09$ & H-29, H-30 $\left({ }^{3} J_{\mathrm{CH}}\right)$ \\
\hline 22 & 37,1 & $1,21,1,43$ & $\mathrm{H}-28\left({ }^{3} J_{\mathrm{CH}}\right)$ \\
\hline 23 & 27,9 & $1,08(\mathrm{~s})$ & H-5, H-24 $\left({ }^{3} J_{\mathrm{CH}}\right)$ \\
\hline 24 & 17,0 & $1,19(\mathrm{~s})$ & H-5, H-23 $\left({ }^{3} J_{\mathrm{CH}}\right)$ \\
\hline 25 & 17,0 & $1,33(\mathrm{~s})$ & $\mathrm{H}-1, \mathrm{H}-5\left({ }^{3} J_{\mathrm{CH}}\right)$ \\
\hline 26 & 18,4 & $1,27(s)$ & H-7 $\left({ }^{3} J_{\mathrm{CH}}\right)$ \\
\hline 27 & 26,0 & $1,11(\mathrm{~s})$ & $\mathrm{H}-15\left({ }^{3} J_{\mathrm{CH}}\right)$ \\
\hline 28 & 28,3 & $0,84(\mathrm{~s})$ & H-18 $\left({ }^{3} J_{\mathrm{CH}}\right)$ \\
\hline 29 & 33,3 & $0,88(\mathrm{~s})$ & H-30 $\left({ }^{3} J_{\mathrm{CH}}\right)$ \\
\hline 30 & 23,7 & $0,88(\mathrm{~s})$ & $\mathrm{H}-29\left({ }^{3} J_{\mathrm{CH}}\right)$ \\
\hline
\end{tabular}

*Os deslocamentos químicos de átomos de hidrogênio sem multiplicidade representam aproximações deduzidas pelos espectros 2D HMQC e HMBC. As constantes de acoplamento $(J$ em $\mathrm{Hz})$ encontram-se entre parênteses. sugeriu a localização da segunda hidroxila no C-6, por comparação com os dados do sumaresinolato de metila $(3 \beta, 6 \beta$-diidroxiolean-28-oato de metila) ${ }^{6}$.

A atribuição do deslocamento químico de $\mathrm{H}-5\left(\mathrm{~m}, \delta_{\mathrm{H}} 0,76\right)$ foi definida no experimento HMQC, pela sua correlação com o sinal em $\delta_{\mathrm{C}} 55,6$ atribuído a C-5, conforme relatado para a maioria dos triterpenóides pentacíclicos ${ }^{6}$. As correlações do sinal de $\mathrm{H}-5\left(\delta_{\mathrm{H}}\right.$ $0,76)$ com os sinais em $\delta_{\mathrm{C}} 79,1$ (C-3), 39,6 (C-4), 68,7 (C-6), 36,4 (C-10) e 27,9 (C-23), entre outras, observadas no espectro HMBC (Tabela 1) confirmaram a localização da segunda hidroxila no C-6.

A configuração $\beta$-axial para a segunda hidroxila, ligada a C-6, foi definida pelo sinal em $\delta 4,58\left(1 \mathrm{H}, \mathrm{sl}, W_{\mathrm{h} / 2}=9 \mathrm{~Hz}\right)$, indicativo de hidrogênio $\alpha$-equatorial e consistente com o relatado na literatura para triterpenóides $6 \beta$-substituídos ${ }^{2,9,10}$. A análise dos dados disponíveis para o triterpenóide 1 permitiu identificá-lo como o $3 \beta, 6 \beta$ diidroxi-olean-12-eno.

O nortriterpenóide 2 não é de ocorrência comum em plantas, tendo sido relatado em Ricinus cummuni, Euphorbia chamaesyce e Claoxylon polot (Euphorbiaceae), em Carlina corymbosa, Chuquiraga ulicina e Koelpinia linearis (Asteraceae), Salvia roborowskii (Lamiaceae) e no líquen Pseudocyphellaria rubella ${ }^{11,12}$. Os dados de RMN ${ }^{13} \mathrm{C}$ para a substância 2 encontram-se descritos na literatura, porém devem ser revisados, sobretudo os relatados por Koul et al. ${ }^{12}$

$\mathrm{O}$ espectro de $\mathrm{RMN}{ }^{1} \mathrm{H}$ de 2 (Tabela 2) apresentou padrão caraterístico de triterpenóides $3 \beta-\mathrm{OH}$, evidenciado pelo duplodubleto em $\delta 3,18(\mathrm{~J}=4,7$ e $11,5 \mathrm{~Hz})$ atribuído ao hidrogênio carbinólico ligado a C-3 e pelos seis singletos entre $\delta$ 0,70-2,20 correspondentes a hidrogênios de grupos metílicos em carbonos não-hidrogenados, sendo que o sinal em $\delta 0,97$ é referente aos hidrogênios de dois grupos $\mathrm{CH}_{3}(\mathrm{H}-23$ e H-27) e o sinal em $\delta$ 2,15 é característico de metila ligada à carbonila.

$\mathrm{O}$ espectro de $\mathrm{RMN}{ }^{13} \mathrm{C} \mathrm{BB}-{ }^{1} \mathrm{HD}$ de 2 (Tabela 2) apresentou 29 sinais que, pelo espectro DEPT $135^{\circ}$, sete são referentes a carbonos de grupos metílicos, dez metilênicos, seis metínicos e seis nãohidrogenados, correspondendo à fórmula molecular $\mathrm{C}_{29} \mathrm{H}_{48} \mathrm{O}_{2} . \mathrm{O}$ sinal em $\delta 78,9(\mathrm{CH})$ é referente ao carbono oximetínico (C-3) e o sinal em 212,9 (C) sugeriu uma carbonila em C-20 no esqueleto lupano $^{7}$, sendo confirmado pelo experimento $\mathrm{HMBC}$ no qual o singleto em $\delta_{\mathrm{H}} 2,15(\mathrm{H}-29)$ correlaciona com $\delta_{\mathrm{C}} 212,9(\mathrm{C}-20)$ e 52,6 (C-19). O triplo-dubleto em $\delta_{\mathrm{H}} 2,57(\mathrm{H}-19)$ correlaciona com os sinais em $\delta_{\mathrm{C}} 27,7(\mathrm{C}-21)$ e $212,9(\mathrm{C}-20)$ e o tripleto em $\delta_{\mathrm{H}} 1,82(\mathrm{H}-$ 18) correlaciona com os sinais em $\delta_{\mathrm{C}} 52,6(\mathrm{C}-19), 212,9(\mathrm{C}-20)$ e 18,0 (C-28).

A análise dos dados de $\mathrm{RMN}{ }^{13} \mathrm{C}$ e comparação com os descritos na literatura para os triterpenóides lupeol ${ }^{6}$ e 30-norlupan-20ona $^{7}$, utilizados como modelos, permitiu identificar a substância 2 como a $3 \beta$-hidroxi-30-norlupan-20-ona.

\section{PARTE EXPERIMENTAL}

\section{Procedimentos experimentais gerais}

Os espectros de RMN ${ }^{1} \mathrm{H} \mathrm{e}{ }^{13} \mathrm{C}$ foram obtidos em espectrômetro Brüker modelo Avance DRX-500, operando a $500 \mathrm{MHz}\left({ }^{1} \mathrm{H}\right)$ e 125 $\mathrm{MHz}\left({ }^{13} \mathrm{C}\right)$. As amostras foram preparadas em tubos de $5 \mathrm{~mm}$ de diâmetro interno, utilizando $\mathrm{CDCl}_{3}$ (Isotec-INC) como solvente e TMS como padrão interno. As placas cromatográficas foram preparadas utilizando uma mistura de gel de sílica $60 \mathrm{G}$ Vetec e 60 $\mathrm{GF}_{254}$ Fluka (1:1) e as revelações das cromatoplacas foram feitas por borrifamento com solução de sulfato cérico. $\mathrm{O}$ critério de pureza adotado para as substâncias isoladas foi a observação de uma única mancha em cromatografia em camada delgada comparativa, 
Tabela 2. Dados de $\mathrm{RMN}{ }^{1} \mathrm{H}(500 \mathrm{MHz})$ e ${ }^{13} \mathrm{C}(125 \mathrm{MHz})$ de $\mathbf{2}$, em $\mathrm{CDCl}_{3}$

\begin{tabular}{|c|c|c|c|}
\hline $\mathrm{C} / \mathrm{H}$ & $\delta_{\mathrm{C}}$ & $* \delta_{\mathrm{H}}$ & $\mathrm{H} \times{ }^{13} \mathrm{C}-\mathrm{HMBC}-{ }^{\mathrm{n}} \boldsymbol{J}_{\mathrm{CH}}(\mathrm{n}=2$ e 3$)$ \\
\hline 1 & 38,7 & $0,90,1,66$ & H-5 $\left({ }^{3} J_{\mathrm{CH}}\right)$ \\
\hline 2 & 27,4 & 1,47 & $-\mathrm{CH}^{\prime}$ \\
\hline 3 & $78,9 ?$ & $18(\mathrm{dd}, \mathrm{J}=4,7,11,5)$ & $\mathrm{H}-23, \mathrm{H}-24\left({ }^{3} J_{\mathrm{CH}}\right)$ \\
\hline 4 & 38,9 & - & H-5, H-23, H-24 $\left({ }^{2} J_{\mathrm{CH}}\right)$ \\
\hline 5 & 55,3 & 0,68 & H-23, H-24, H-25 $\left({ }^{3} J_{\mathrm{CH}}\right)$ \\
\hline 6 & 18,3 & $1,38,1,53$ & H-5 $\left({ }^{2} J_{\mathrm{CH}}\right)$ \\
\hline 7 & 34,2 & 1,39 & $\mathrm{H}-26\left({ }^{3} J_{\mathrm{CH}}\right)$ \\
\hline 8 & 40,7 & - & $\mathrm{H}-26\left({ }^{2} J_{\mathrm{CH}}\right) ; \mathrm{H}-27\left({ }^{3} J_{\mathrm{CH}}\right)$ \\
\hline 9 & 50,3 & 1,25 & $\mathrm{H}-25, \mathrm{H}-26\left({ }^{3} J_{\mathrm{CH}}\right)$ \\
\hline 10 & 37,2 & - & H-25 $\left({ }^{2} J_{\mathrm{CH}}\right)$ \\
\hline 11 & 20,9 & $1,24,1,43$ & - \\
\hline 12 & 27,2 & 1,04 & - \\
\hline 13 & 37,0 & 1,57 & $\mathrm{H}-18\left({ }^{2} J_{\mathrm{CH}}\right)$ \\
\hline 14 & 42,7 & - & H-27 $\left({ }^{2} J_{\mathrm{CH}}\right) ; \mathrm{H}-26\left({ }^{3} J_{\mathrm{CH}}\right)$ \\
\hline 15 & 27,3 & 1,64 & $\mathrm{H}-27\left({ }^{3} J_{\mathrm{CH}}\right)$ \\
\hline 16 & 35,0 & $1,44,1,50$ & $\mathrm{H}-28\left({ }^{3} J_{\mathrm{CH}}\right)$ \\
\hline 17 & 43,1 & - & $\mathrm{H}-18, \mathrm{H}-28\left({ }^{2} J_{\mathrm{CH}}\right)$ \\
\hline 18 & 50,7 & $1,82(\mathrm{t}, \mathrm{J}=11,4)$ & $\mathrm{H}-28\left({ }^{3} J_{\mathrm{CH}}\right)$ \\
\hline 19 & 52,6 & $\begin{array}{c}2,57(\mathrm{td}, \mathrm{J}=5,3 \\
11,3)\end{array}$ & H-28 $\left({ }^{2} J_{\mathrm{CH}}\right) ; \mathrm{H}-29\left({ }^{3} J_{\mathrm{CH}}\right)$ \\
\hline 20 & 212,9 & - & $\begin{array}{c}\mathrm{H}-19, \mathrm{H}-29\left({ }^{2} J_{\mathrm{CH}}\right) ; \mathrm{H}-18, \\
\mathrm{H}-21\left({ }^{3} J_{\mathrm{CH}}\right)\end{array}$ \\
\hline 21 & 27,7 & $2,03(\mathrm{~m})$ & H-19 $\left({ }^{2} J_{\mathrm{CH}}\right)$ \\
\hline 22 & 39,8 & $1,35,1,46$ & $\mathrm{H}-28\left({ }^{3} J_{\mathrm{CH}}\right)$ \\
\hline 23 & 28,0 & 0,97 (s) & $\mathrm{H}-24\left({ }^{3} J_{\mathrm{CH}}\right)$ \\
\hline 24 & 15,4 & $0,76(s)$ & H-3 $\left({ }^{3} J_{\mathrm{CH}}\right)$ \\
\hline 25 & 16,1 & $0,83(\mathrm{~s})$ & - \\
\hline 26 & 15,9 & $1,02(\mathrm{~s})$ & - \\
\hline 27 & 14,5 & $0,97(s)$ & - \\
\hline 28 & 18,0 & 0,77 (s) & H-18 $\left({ }^{3} J_{\mathrm{CH}}\right)$ \\
\hline 29 & 29,2 & $2,15(\mathrm{~s})$ & - \\
\hline
\end{tabular}

*Os deslocamentos químicos de átomos de hidrogênio sem multiplicidade representam aproximações deduzidas pelos espectros 2D HMQC e HMBC. As constantes de acoplamento $(J$ em Hz) encontram-se entre parênteses.

em diferentes sistemas de eluentes. As colunas cromatográficas foram feitas à pressão atmosférica utilizando gel de sílica $60(0,2-$ 0,5 mm) da Merck (7733) e Sephadex LH-20 da Sigma (9041-376), sendo este último previamente reciclado quando necessário.

\section{Material vegetal}

O material vegetal foi coletado no município de Nazaré do Piauí - PI, Brasil, em agosto de 1998. A identificação foi realizada no Herbário Graziela Barroso - UFPI, onde se encontra depositada uma exsicata da espécie, sob o número 10340.

\section{Isolamento dos constituintes}

As folhas de T. brasiliensis (1,3 kg) foram secas, moídas e extraídas por maceração com etanol à temperatura ambiente. $\mathrm{O}$ extrato etanólico foi parcialmente concentrado em evaporador rotatório, sob pressão reduzida a um volume de aproximadamente $400 \mathrm{~mL}$, adicionando-se $800 \mathrm{~mL}$ de água e, em seguida, extraído com acetato de etila. A fase acetato de etila foi concentrada, dissolvida em $500 \mathrm{~mL}$ de uma solução de metanol-água (9:1) e extraída com hexano, fornecendo as fases hexânica e hidroalcoólica (32 g).
A fase hexânica concentrada foi dissolvida em metanol a quente (banho de água a $40{ }^{\circ} \mathrm{C}$ ), mantida sob refrigeração por $24 \mathrm{~h}$ e filtrada, resultando em duas frações, sendo uma solúvel em hexano (4 g) e outra, solúvel em metanol (4 g). O material solúvel em $\mathrm{MeOH}$ foi fracionado através de cromatografia em coluna de gel de sílica, eluída com hexano-AcOEt em gradiente de polaridade crescente, fornecendo 115 frações (125 mL cada) que, depois de concentradas, foram analisadas por CCDC e reunidas em 26 grupos.

Entre as frações eluídas com hexano, o grupo D1 (frações 1-3) foi recromatografado em coluna de Sephadex LH-20 com hexanodiclorometano (1:4) e forneceu a substância 6 (21 mg).

A fração D9 (554 mg) e os grupos D11 (frações 11-16) e D19 (frações 19-26) foram eluídos com hexano-AcOEt (95:5). D9 foi sucessivamente recromatografada em coluna de Sephadex LH-20 com hexano-diclorometano (1:4) fornecendo 8 (21 mg) e a fração D9-6. Esta última foi recromatografada em coluna de gel de sílica com hexano-diclorometano em ordem crescente de polaridade fornecendo 3c e a mistura dos triterpenóides 3a, 3b e 3c. Os grupos D19 e D11 foram recristalizados, o primeiro em metanol e o segundo em hexano, fornecendo, respectivamente, a substância 7 (47 $\mathrm{mg}$ ) e $300 \mathrm{mg}$ da mistura de $\mathbf{4 a}, \mathbf{4 b}$ e $\mathbf{4 c}$.

Os grupos D40 (frações 40-44) e D45 (frações 45-47) foram eluídos com hexano-AcOEt (9:1). D40 foi suspenso em hexano originando um sólido que, separado por filtração, forneceu a substância 5c (22 mg) e o sobrenadante foi recromatografado em coluna de Sephadex LH-20, eluído com hexano-diclorometano (1:4), fornecendo 1 ( $6 \mathrm{mg}$ ). O grupo D45 foi recromatografado em coluna de Sephadex LH-20 eluído com hexano-diclorometano (1:4), fornecendo 2 (7 mg) e $9 \mathrm{mg}$ da mistura de $\mathbf{5 a}, \mathbf{5 b}$ e $\mathbf{5 c}$.

As substâncias isoladas foram identificadas por espectrometria de RMN ${ }^{1} \mathrm{H}$ e ${ }^{13} \mathrm{C}$ e seus dados mostraram-se de acordo com os descritos na literatura. Para os triterpenóides $\mathbf{1}$ e $\mathbf{2}$ foram utilizados modelos e dados dos experimentos bidimensionais (HMQC e HMBC).

\section{CONCLUSÃO}

O fracionamento do material solúvel em $\mathrm{MeOH}$, proveniente da partição do extrato EtOH de folhas, através de colunas de gel de sílica e de Sephadex LH-20, conduziu ao isolamento de 12 triterpenóides. Com exceção do esqualeno, todos são pentacíclicos de esqueletos tipo lupano, oleanano e ursano. O componente majoritário das três misturas ternárias de triterpenos é de esqueleto lupano. Este padrão de misturas ternárias não foi observado em nenhuma espécie do gênero Terminalia.

$\mathrm{O}$ triterpenóide $3 \beta, 6 \beta$-diidroxi-olean-12-eno (daturadiol) e o nortriterpenóide 3 $\beta$-hidroxi-30-norlupan-20-ona são inéditos na família Combretaceae. O primeiro encontra-se relatado somente em Solanaceae e o segundo em Euphorbiaceae, Asteraceae e Lamiaceae, bem como no líquen Pseudocyphellaria rubella.

\section{AGRADECIMENTOS}

Ao CNPq e à CAPES pela bolsa de mestrado (D. S. Araújo) e apoio financeiro (PROCAD No 0014/01-0), à Dra. G. M. Sousa do Herbário Graziela Barroso, UFPI, pela coleta e identificação do material botânico, ao Centro Nordestino de Aplicação e Uso da Ressonância Magnética Nuclear (CENAUREMN-UFC) pela execução dos espectros.

\section{REFERÊNCIAS}

1. Katerere, D. R.; Gray, A. I.; Nash, R. J.; Waigh, R. D.; Phytochemistry 2003 63, 81; Saleem, A.; Husheem, M.; Härkönen, P.; Pihlaja, K.; J. Ethnopharmacol. 2002, 81, 327. 
2. Garcez, F. R.; Garcez, W. S.; Miguel, D. L. S.; Serea, A. A. T.; Prado, F. C.; J. Braz. Chem. Soc. 2003, 14, 461.

3. Carpano, S. M.; Spegazzini, E. D.; Rossi, J. S.; Castro, M. T.; Debenedetti, S. L.; Fitoterapia 2003, 74, 294; Khan, M. R.; Kihara, M.; Omoloso, A D.; Fitoterapia 2002, 73, 737; Mau, J.-L.; Ko, P.-T.; Chyau, C.-C.; Food Res. Int. 2003, 36, 97; Rao, B. K.; Sudarshan, P. R.; Rajasekhar, M. D.; Nagaraju, N.; Rao, C. A.; J. Ethnopharmacol. 2003, 85, 169; Valsaraj, R.; Pushpangadan, P.; Smitt, U. W.; Adsersen, A.; Christensen, S. B.; Sittie, A.; Nyman, U.; Nielsen, C.; Olsen, C. E.; J. Nat. Prod. 1997, 60, 739.

4. Corrêa, M. P.; Dicionário das Plantas Úteis do Brasil, Impressa Nacional: Rio de Janeiro, 1974, vol. 5, p. 195.

5. Olea, R. S. G.; Roque, N. F.; Quim. Nova 1990, 13, 278.

6. Mahato, S. B.; Kundu, A. P.; Phytochemistry 1994, 37, 1517.

7. Wenkert, E.; Baddeley, G. V.; Burfitt, I. R.; Moreno, L. N.; Org. Magn. Reson. 1978, 11, 337.

8. Núñez, C. V.; Dissertação de Mestrado, Universidade de São Paulo, Brasil, 1996; Breitmaier, E.; Voelter, W.; Carbon-13 NMR spectroscopy: highresolution methods and applications in Organic Chemistry and Biochemistry, $3^{\text {rd }}$ ed., VCH: New York, 1987, p. 327; Matsuo, M.; Urano, S.; Tetrahedron 1976, 32, 229; Pauletti, P. M.; Araújo, A. R.; Bolzani, V. S.; Quim. Nova 2002, 25, 349.
9. Kocor, M.; Pyrek, J. S.; Atal, C. K.; Bedi, K. L.; Sharma, B. R.; J. Org Chem. 1973, 38, 3685; Itoh, T.; Tamura, T.; Matsumoto, T.; Phytochemistry 1977, 16, 1723; Tori, M.; Takai, M.; Matsumoto, Y.; Moriyama, Y.; Tsuyuki, T.; Takahashi, T.; Ohnishi, H.; Itai, A.; Iitaka, Y.; Bull. Chem. Soc. Jpn. 1984, 57, 2490.

10. Ngounou, F. N.; Lontsi, D.; Sondengam, B. L.; Phytochemistry 1988, 27, 301 .

11. Thompson, M. J.; Bowers, W. S.; Phytochemistry 1968, 7, 845; Hui, W. H.; Li, M. M.; Lee, Y. C.; Phytochemistry 1977, 16, 607; Flagg, M. L.; Valcic, S.; Montenegro, G.; Gomez, M.; Timmermann, B. N.; Phytochemistry 1999, 52, 1345; Tanaka, R.; Ida, T.; Takaoka, Y.; Kita, S.; Kamisako, W.; Matsunagat, S.; Phytochemistry 1994, 36, 129; Corbett, R. E.; Cong, A. N. T.; Holland, P. T.; Wilkins, A. L.; Aust. J. Chem. 1987, $40,461$.

12. Li, Y.; Wu, Y.-Q.; Shi, Y.-P.; Pharmazie 2003, 58, 937; Koul, S.; Razdan, T. K.; Andotra, C. S.; Kalla, A. K.; Koul, S.; Taneja, S. C.; Dhar, K. L.; Phytochemistry 2000, 53, 305 . 